Pacific Journal of Mathematic 


\title{
ON A THEOREM OF PLANCHEREL AND PÓLYA
}

\author{
R. M. REDHEFFER
}

1. Introduction. Paley and Wiener [6] have shown that the following classes of entire functions are equivalent:

(A) those which are $o\left(e^{a|z|}\right)$ in the whole plane and belong to $L^{2}$ on the real axis;

(B) those which can be represented in the form

$$
F(z)=\int_{-a}^{a} e^{i z t} f(t) d t
$$

with $f(t) \in L^{2}$ on $[-a, a]$.

A simple proof was given later by Plancherel and Pólya [7], and they showed how the condition $o\left(e^{a|z|}\right)$ could be weakened in the passage from (A) to (B). Their result leads at once to the following, which is the form to be used in the present discussion:

THEOREM A (Plancherel and Pólya). Let $F(z)$ be an entire function of order 1 , type a. If $F(x) \in L^{2}$ on $(-\infty, \infty)$ then $F(z)$ can be represented in the form

$$
F(z)=\int_{-a}^{a} e^{i z t} f(t) d t
$$

with $f(t) \in L^{2}$ on $[-a, a]$.

The hypothesis concerning order and type means

$$
\lim \sup \log |F(z)| /|z| \leq a, \quad|z| \rightarrow \infty .
$$

Theorem A implies a nontrivial result about entire functions; namely, if $F(z)$ satisfies (1) and is in $L^{2}$ on the real axis, then [ 7 ] 


$$
F(z)=o\left(e^{a r|\sin \theta|}\right), \quad z=r e^{i \theta} .
$$

We shall show here how Theorem A can be used to give very simple proofs of other results, some of which seem accessible only with more difficulty to purely complex-variable methods.

2. The growth of $F(z)$. The Plancherel-Pólya result determines the growth of $F(z)$ in the whole plane from the growth on the real axis:

THE OREM 1. Let $F(z)$ be an entire function satisfying (1), such that

$$
F(x)=O\left(|x|^{n}\right)
$$

for some positive or negative integer $n$, as $x \longrightarrow \infty$ on the real axis. Then

$$
F\left(r e^{i \theta}\right)=O\left(r^{n} e^{a r|\sin \theta|}\right)
$$

uniformly in $\theta$, as $r \rightarrow \infty$.

THEOREM 2. Let $F(z)$ be an entire function satisfying (1), such that

$$
|F(x)| \leq A
$$

for all real $x$. Then

$$
|F(x+i y)| \leq A e^{a|y|}
$$

in the whole plane. If $\lambda=p+i q$ is a zero of $F(z)$, then

$$
|F(z)| \leq A e^{a|y|}|z-y| /|q| \text {. }
$$

These results (which are probably well known) can be obtained at once by [8]; for example, applying [8] to $F(i z) e^{-a z} /\left(A z^{n}+B\right)$ gives Theorem 1 when $n \geq 0$. Since our primary purpose here is to illustrate a method, however, we deduce them from Theorem A. Assume that $F(z)$ in Theorem 2 has a complex zero $\lambda=p+i q, q \neq 0$. (In the contrary case consider $F(z)(z-\lambda-i q) /(z-\lambda)$, where $\lambda$ is a real zero, and let $q \rightarrow 0$.) We have

$$
G(z)=[F(z)]^{m} /(z-\lambda)=\frac{1}{\sqrt{2 \pi}} \int_{-m a}^{m a} f(t) e^{i z t} d t, f(t) \in L^{2},
$$

where $m$ is an integer. (A similar use of the $m^{\text {th }}$ power of a function is made in [5] and [7].) By a short calculation, we get 


$$
\int_{-m a}^{m a}|f(t)|^{2} d t=\int_{-\infty}^{\infty}|G(t)|^{2} d x \leq A^{m} \pi /|q|
$$

so that, by the Schwartz inequality in (1),

$$
|G(z)|^{2} \leq \frac{1}{\sqrt{2 \pi}}\left(\int_{-m a}^{m a} e^{2|y| t} d t\right)\left(A^{2 m} \pi /|q|\right), \quad z=x+i y .
$$

Hence

$$
|F(t)|^{2 m} \leq|z-\lambda|^{2} C A^{2 m} e^{2 m a|y|},
$$

where $C$ is constant. Taking the $m^{\text {th }}$ root and letting $m \rightarrow \infty$ completes the proof. The proof of Theorem 1 is similar, if we define

$$
G(z)=(z-\lambda)^{-1}[F(z) / p(z)]^{m},
$$

where $p(z)$ is a polynomial of degree $n$ formed from the zeros, other than $\lambda$, of $F(z)$.

The second part of Theorem 2 results when we apply the first part to $F(z) /$ $(z-\lambda)$; it could be sharpened by including more zeros. As it stands, however, this second part already gives the following:

Corolla ry. Let $F(z)$ satisfy the hypothesis of Theorem 2, and suppose

$$
F\left(r e^{i \theta}\right) \sim A e^{a r|\sin \theta|}
$$

for a particular $\theta$, as $r \longrightarrow \infty$. Then at most a finite number of zeros $\lambda$ satisfy $\pi+\theta-\delta>2 \arg \lambda>\theta+\delta$ for any positive $\delta$.

3. Complex roots. A consequence of Theorem 1 is:

THEOREM 3. Let $F(z)$ satisfy the hypothesis of Theorem 1 , and let $n(x)$ denote the number of real roots of the equation $F(z)=0$ which lie in the circle $|z| \leq x$. If

$$
\underset{r \rightarrow \infty}{\limsup } \int_{1}^{r} n(x) d x / x-2 a r / \pi+b \log r>-\infty,
$$

then the equation $F(z)=0$ has at most $b+n$ complex roots in the whole plane.

The proof is practically contained in a discussion of Levinson [5]. If $N(x)$ denotes the number of roots of $F(z)=0$ in the circle $|z| \leq x$, Jensen's theorem 
combines with the conclusion of Theorem 1 to give

$$
\begin{aligned}
\int_{1}^{r} N(x) d x / x-A & =\frac{1}{2 \pi} \int_{0}^{2 \pi} \log \left|F\left(r e^{i \theta}\right)\right| d \theta \\
& \leq \frac{1}{2 \pi} \int_{0}^{2 \pi}(n \log r+a r|\sin \theta|) d \theta+B \\
& =n \log r+2 a r / \pi+B,
\end{aligned}
$$

where $A$ and $B$ are constants. Hence the number of complex zeros,

$$
c(x)=N(x)-n(x),
$$

satisfies

$$
\int_{1}^{r} c(x) d x / x \leq(n+b) \log r+C
$$

for some arbitrarily large $r$ 's, where $C$ is constant. It follows that $c(x) \leq n+b$, as was to be shown.

By means of the following result, Duffin and Schaeffer have given simple proofs, and improvements, of some theorems due to Szegö, Bernstein and Boas ( see below):

THEOREM 4 (Duffin and Schaeffer). Let $F(z)$ be an entire function such that

$$
F(z)=O\left(e^{a|z|}\right)
$$

If $F(x)$ is real for all real $x$ and satisfies $|F(x)| \leq A$, then the equation

$$
F(z)=A \cos (a z+B)
$$

has no complex roots.

Theorem 3 contains Theorem 4, and in fact gives a slight generalization of it:

THЕОRЕM 5. Let $F(z)$ be an entire function satisfying (1). If $F(x)$ is real for real $x$ and satisfies

$$
|F(x)| \leq|P(x)|
$$


where $P(x)$ is a real polynomial of degree $n$, then the equation

$$
F(z)=P(z) \cos (a z+B)
$$

has at most $n+1$ complex roots.

A linear change of variable enables us to assume $a=\pi, B=0$. Since $F(z)$ $P(z) \cos \pi z$ is nonpositive when $\cos \pi z=1$, and nonnegative when $\cos \pi z=-1$, the equation

$$
F(z)=P(z) \cos \pi z
$$

has a root in every interval $m \leq z \leq m+1$, where $m$ is an integer (cf. [3]). Any root occurring at the ends of these intervals is multiple. Hence if $n(x)$ is the number of real zeros $\lambda$ satisfying $|\lambda| \leq x$, then $n(x)$ is at least equal to the function $n_{1}(x)$, defined as 0 for $0 \leq x \leq 1$, as 2 for $1<n \leq 2$, and so on. A short calculation gives

$$
\int_{1}^{n+} n(x) d x / x \geq \int_{1}^{n+} n_{1}(x) d x / x=2 \log \left(n^{n} / n !\right) \sim 2 n-\log n,
$$

so that Theorem 5 follows from Theorem 3 with $b=1$. Since complex zeros occur in pairs, Theorem 5 contains Theorem 4.

According to Paley and Wiener [6], a set of functions $\left\{e^{i \lambda_{n} x}\right\}$ has deficiency $d$ on a given closed interval if it becomes complete in $L^{2}$ when $d$ but not fewer functions $\left\{e^{i \lambda x}\right\}$ are adjoined to the set. Similarly, the set has excess $e$ if it remains complete when $e$ terms, but not more, are removed. Here we adopt the convention that a negative deficiency $d$ means an excess $-d$. That the deficiency $d$ is well defined follows from a theorem of Levinson [5]:

THEOREM 6 (Levinson). If the set $\left\{e^{i \lambda_{n} x}\right\}$ is complete $L^{p}$ on a finite interval, it remains complete when any $\lambda_{n}$ is changed to another number.

The result remains true even when several $\lambda$ 's are equal, if we agree to require a zero of the corresponding multiplicity in the entire function

$$
F(z)=\int_{-a}^{a} e^{i z t} f(t) d t, \quad f \in L^{p},
$$

which vanishes at the $\lambda_{n}$ 's. In this setting, the previous theorems concerning zeros appear as special cases of the following:

THEOREM 7. Let $F(z)$ be an entire function satisfying (1), and suppose 


$$
F(x)=O\left(|x|^{n}\right)
$$

on the real axis. If $F(z)=0$ at a set $\left\{\lambda_{n}\right\}$ such that $\left\{e^{i \lambda_{n} x}\right\}$ has deficiency $d$ on an interval of length $2 \pi a$, then $F(z)$ has at most $d+n$ zeros other than the $\lambda_{n}$ 's.

The truth of the assertion is evident from

$$
\left((z) F(z) / P(z)=\int_{-a}^{a} f(t) e^{i z t} d t, \quad f(t) \in L^{2},\right.
$$

where $Q(z)$ is any polynomial of degree $d$, and $P(x)$ is a polynomial of degree $d+n+1$ formed from the (supposed) extra zeros of $F(z)$. That the result contains The orem 5 and hence Theorem 6 follows from a theorem of Levinson [5] to the effect that $\left\{e^{i \lambda_{n} x}\right\}$ has deficiency at most $d$ on $[0,2 \pi]$ if

$$
\left|\lambda_{n}\right| \leq|n|+d / 2+1 / 4, \quad-\infty<n<\infty
$$

(cf. also [6]).

4. Completeness. Pursuing the subject of completeness in more detail, we find that some of Paley and Wiener's work can be simplified and generalized by use of Theorem A (cf. Theorems XXIX and XXX of [6]).

THEOREM 8. Let $\left\{\lambda_{n}\right\}$ be a set of complex numbers such that the set $\left\{e^{ \pm i \lambda_{n} x}\right\}$ has finite (positive, zero or negative) deficiency on some finite interval. Then the deficiency is $d$ if and only if

$$
\int_{1}^{\infty} x^{2 d-2}|F(x)|^{2} d x<\infty, \int_{1}^{\infty} x^{2 d}|F(x)|^{2} d x=\infty
$$

where

$$
F(z)=\prod\left(1-z^{2} / \lambda_{n}^{2}\right)
$$

We confine our attention to the case $d=1$, since the general case is reduced to that by considering $P(z) F(z)$ or $F(z) / P(z)$ as heretofore. Suppose, then, that the set has deficiency $d=1$ on an interval of length $2 a$. Since the set is not complete, there is a function $G(z)$,

$$
G(z)=\int_{-a}^{a} f(t) e^{i z t} d t, \quad f(t) \in L^{2},
$$

such that $G\left(\lambda_{n}\right)=0$. By the Hadamard factorization theorem (cf. also [5]) we 
have

$$
G(z)=F(z) e^{b z} P(z)
$$

where $P(z)$ is a polynomial. Now actually $P(z)$ is constant, since otherwise $G(z)$ would have an extra zero, and the deficiency of the original set would be greater than 1 . Hence (9) gives

$$
F(z)=e^{-b z} G(z) C,
$$

where $C$ is constant. If $b$ has positive real part, then (10) shows that $F(x)$ decreases exponentially as $x \longrightarrow \infty$. Since $F$ is even, the same is true as $x \rightarrow-\infty$, and hence $F(z) \equiv 0$ by a well-known result of Carlson. Similarly if $b$ has negative real part. It follows that $b$ is pure imaginary, so that

$$
F(x)=\int_{-a}^{a} f(t) e^{i(x+c) t} d t, \quad c \text { real, }
$$

and hence $F(x) \in L^{2}$ by the Plancherel theorem.

On the other hand, if $x F(x) \in L^{2}$ then Theorem A yields the representation

$$
z F(z)=\int_{-a}^{a} f(t) e^{i z t} d t
$$

since ( 11 ) ensures ( 1 ); and hence the deficiency exceeds 1 .

Suppose next that the deficiency is an unknown but finite number, and that

$$
\int_{1}^{\infty}|F(x)|^{2} d x<\infty, \quad \int_{1}^{\infty} x^{2}|F(x)|^{2} d x=\infty
$$

With $2 a$ as the interval of completeness, there is a function $G(z)$,

$$
G(z)=\int_{-a}^{a} f(t) e^{i z t} d t, \quad f(t) \in L^{2},
$$

such that $G(z)=0$ at all but a finite number, say $n$, of the $\lambda$ 's, and has no other zeros. (Otherwise the set would have infinite negative deficiency). The Hadamard theorem gives

$$
F(z)=e^{b z} P(z) G(z),
$$

where $P(z)$ is a polynomial. If the imaginary part of $b=p+i q$ is positive, then 


$$
\lim \sup \log |F(i y) / y| \leq a-q \text { as } y \rightarrow \infty \text {, }
$$

and hence the same is true as $y \longrightarrow-\infty$. Similarly if the imaginary part is negative. In either case, then, $F(z)$ satisfies (1). Equation (12) now combines with Theorem A to show that

$$
F(z)=\int_{-a}^{a} g(t) e^{i z t} d t, \quad g(t) \in L^{2}
$$

so that the set $\left\{e^{i \lambda_{n} x}\right\}$ is not complete. Thus the deficiency is at least 1 .

On the other hand, if the deficiency is $n>1$ then the Hadamard theorem, as before, gives

$$
P(z) F(z) e^{b z}=\int_{-a}^{a} f(t) e^{i z t} d t, \quad f(t) \in L^{2}
$$

where $P(z)$ is a polynomial of degree $n-1$. As before, the presence of $b$ causes no difficulty, so that $P(x) F(x) \in L^{2}$. This contradicts (12).

Theorem 8 contains Theorem 6 for the case $L^{2}$, although Levinson's general case $L^{p}$ seems somewhat deeper. We give an application:

THEOREM 9. Let

$$
F(z)=\prod\left(1-z^{2} / \lambda_{n}^{2}\right)
$$

where the $\lambda_{n}$ are complex numbers, and let the equation $F(x)=A$ have roots $\lambda_{n}^{\prime}$, where $A$ is a complex nonzero constant. If $\left\{e^{i \lambda_{n} x}\right\}$ has finite deficiency $d$ and $\left\{e^{i \lambda_{n}^{\prime} x}\right\}$ has finite deficiency $d^{\prime}$, then $d<0$ implies $d^{\prime}=d$, and $d>0$ implies $d^{\prime}=0$. If $d=0$ then $d^{\prime} \geq 0$.

It should be observed that $d^{\prime}$ is restricted to be finite in the hypothesis of the theorem, and only then can we evaluate $d^{\prime}$ more exactly. With regard to this assumption, the following may be said. First, the set $\exp \left(i \lambda_{n}^{\prime} x\right)$ cannot have infinite excess; that is, $d^{\prime} \neq-\infty$. In the other direction, the set is complete on every interval of length less than the interval for $\left\{\lambda_{n}\right\}$ (which does not mean, however, that $d^{\prime}$ is finite). For the case of real $\lambda_{n}$, an elementary but long argument shows that in fact $d^{\prime}$ is finite, so that we can then dispense with this extra hypothesis. These matters lie to one side of the present discussion, since their proof does not involve Theorem A, and we omit them. 
A second remark may be in order. It is well known that all the $A$-points of a canonical product have the same exponent of convergence, and in Theorem 9 one can prove the stronger result that $\lim \Lambda(u) / u$ and $\lim \Lambda^{\prime}(u) / u$ both exist and are equal. Even this statement is less precise than the conclusion of the theorem, however. It is easy to construct sets with equal density, such that one set has infinite excess and the other has infinite deficiency on a given interval. We conjecture, incidentally, that one can make $d=0, d^{\prime}=m$, where $m$ is any positive integer, so that the nebulous case $d=0$ cannot be improved.

To establish Theorem 9, write

$$
\int_{1}^{\infty}|F(x)-A|^{2} x^{2 d-2} \leq \int|F|^{2} x^{2 d-2}+2|A| \int|F| x^{2 d-2}+|A|^{2} \int x^{2 d-2}
$$

which is finite if $d \leq 0$, by The orem 8 and the Schwartz inequality applied to the second integral. Hence, by Theorem 8 again,

$$
d^{\prime} \geq d \text { if } d \leq 0
$$

Writing

$$
F(z)=[F(z)-a]+a,
$$

and turning the argument about, gives

$$
d \geq d^{\prime} \text { if } d^{\prime} \leq 0 .
$$

Suppose now $d>0$, so that, by Theorem 8 ,

$$
\int_{1}^{\infty}|F(x)|^{2} d x<\infty
$$

This implies $F(x) \longrightarrow 0$, as is well known, so that $F(x)-A$ is dominated by $A$. Hence by Theorem 8 the zeros form an exact set:

$$
d^{\prime}=0 \text { if } d>0
$$

Similarly,

$$
d=0 \text { if } d^{\prime}>0
$$

Equations (13) and (16) show that $d<0$ implies $0>d^{\prime}>d$. But then (14) gives $d \geq d^{\prime}$, since $d^{\prime} \leq 0$; and thus $d<0$ implies $d^{\prime}=d$. 
5. An inequality for entire functions. In a series of interesting papers [2], [3], [4], Duffin and Schaeffer establish some inequalities for entire functions of exponential type bounded on the real axis. From these they obtain, sometimes in sharpened form, the classical inequalities of Bernstein and others for bounded polynomials. The main results are as follows:

THEOREM 10 (Duffin and Schaeffer). Let $F(z)$ be an entire function, real on the real axis, which satisfies

$$
F(z)=O\left(e^{a|z|}\right)
$$

in the whole plane and $|F(x)| \leq 1$ for $-\infty<x<\infty$. Then, with $z=x+i y$, we have

$$
|F(z)| \leq \cosh a y,|F(z)|^{2}+\left|F^{\prime}(z)\right|^{2} / a^{2} \leq \cosh 2 a y
$$

If there is equality at any point except points on the real axis where $F(x)= \pm 1$, then $F(x)=\cos (b x+c)$.

Our Theorem 1 shows that the hypothesis $O\left(a^{a|z|}\right)$ can be replaced by (1). The procedure in [2] is to deduce the result for $y=0$ first, by means of Theorem 4. In this form the statement seems due chiefly to Boas [1]:

THE OREM 11 (Duffin, Schaeffer, and Boas). Let $F(z)$ be an entire function satisfying (1) and real on the real axis. If $|F(x)| \leq 1$ for all real $x$ then

$$
|F(x)|^{2}+\left|F^{\prime}(x)\right|^{2} / a^{2} \leq 1
$$

for all real $x$.

A modification of Duffin and Schaeffer's argument ${ }^{1}$ enables us to deduce Theorem 11 from Theorem A. Suppose the hypothesis fulfilled, but let the conclusion be violated at a particular point $x=b$. By considering $\pm F( \pm z / a)$, we may assume

$$
F(b) \geq 0, F^{\prime}(b) \leq 0 \text {, and } a=1 \text {, }
$$

besides

$$
|F(b)|^{2}+\left|F^{\prime}(b)\right|^{2}>1
$$

The equation $F(b)=\cos z$ has a root $z=r, 0 \leq r \leq \pi / 2$, since $1 \geq F(a) \geq 0$.

\footnotetext{
${ }^{1}$ The author regrets having presented this discussion to the American Mathematical Society without knowing of Duffin and Schaeffer's work.
} 
Now, in fact $r>0$. For if $r=0$ then $F(b)=1$, and (2) yields $F^{\prime}(b)<0$. Hence $F(x)$ is strictly decreasing at $x=b$, so that $F(x)>1$ for some $x<b$.

If we define

$$
G(z)=F(z+b-r)-\cos z
$$

then

$$
G(r)=0, G(0) \leq 0, G(\pi) \geq 0
$$

Moreover,

$$
G^{\prime}(r)=F^{\prime}(b)+\sin r=F^{\prime}(b)+\left[1-F^{2}(b)\right]^{1 / 2}<0,
$$

the last inequality being a consequence of $F^{\prime}(b) \leq 0$ and (17). Combined with (19), the condition $G^{\prime}(r)<0$ shows readily that $G(z)=0$ has three roots $r_{0}<$ $r<r_{1}$ in the interval $0 \leq z \leq \pi$; and if $r_{0}=0$ or $r_{1}=\pi$ the corresponding root is multiple, since $|F(x)| \leq 1$. Besides these roots, $G(z)=0$ has roots $r_{n}$ in each interval $[n \pi,(n+1) \pi], n= \pm 1, \pm 2, \cdots$. Thus, the function

$$
H(z)=G(z) /(z-r)=\int_{-1}^{1} e^{i z t} h(t) d t, \quad h(t) \in L^{2},
$$

has roots at $r_{0}$ and $r_{n}, n= \pm 1, \pm 2, \cdots$, where the enumeration can be so managed that

$$
\left|r_{n}\right| \leq|n| \pi
$$

By Levinson's theorem cited above, the set $\left\{e^{i r_{n} x}\right\}$ is complete $L^{2}$ on $[-1,1]$, and therefore $h(t)=0$ almost everywhere. If the inequality of Theorem 11 becomes an equality at a point where $F(x) \neq \pm 1$, then the corresponding root of $G(z)$ is easily seen to be triple, so that the same discussion holds

6. Differences and derivatives. We conclude with a theorem of different type, concerning classes of functions:

THEOREM 12. Let $C$ denote the class of entire functions which satisfy (1) and belong to $L^{2}$ on the real axis. Let $h$ be any complex or real nonzero number, except that $|h|<2 \pi / a$ if $h$ is real. Then the class of functions $F^{\prime}(z)$, where $F$ ranges over $C$, is identical with the class of functions $G(z+h)-G(z)$, where $G$ ranges over $C$. But if $h$ is real and $|h| \geq 2 \pi / a$, the latter class is always $a$ proper subset of the former. 
Results of the same sort without $L^{2}$ condition are well known; for example, Carmichael has shown that the equation

$$
F(z+1)-F(z)=G(z)
$$

has a unique solution of type $a$ on the real axis and $c$ on the imaginary axis, if $G(z)$ is of this same type, $G(0)=1$, and $c<\pi$. To prove Theorem 12 , let $F(z)$ be in $C$, so that by Theorem $\mathrm{A}$ we have

$$
\begin{aligned}
F(z+h)-F(z) & =\int_{-a}^{a} e^{i z t}\left(e^{i h t}-1\right) f(t) d t, \quad f \in L^{2} \\
& =\int_{-a}^{a} e^{i z t}\left[\left(e^{i h t}-1\right) / i t\right] f(t) i t d t \\
& =\int_{-a}^{a} e^{i z t} i t g(t) d t, \quad g \in L^{2} .
\end{aligned}
$$

Hence, every function of the form $F(z+h)-F(z)$, with $F \in C$, is representable as $G^{\prime}(t)$ with $G \in C$. Similarly, let $G \in C$, so that

$$
\begin{aligned}
G^{\prime}(z) & =\int_{-a}^{a} i t e^{i z t} g(t) d t, \quad g \in L^{2} \\
& =\int_{-a}^{a} e^{i z t} \frac{i t}{e^{i h t}-1}\left(e^{i h t}-1\right) g(t) d t(h t \neq 2 n \pi) \\
& =\int_{-a}^{a} e^{i z t}\left(e^{i h t}-1\right) f(t) d t, \quad f(t) \in L^{2} .
\end{aligned}
$$

Thus $G^{\prime}(z)$ is representable as $F(z+h)-F(z)$ with $F \in C$, provided $h t \neq 2 n \pi$ for $-a \leq t \leq a$. The latter condition is fulfilled unless $h$ is real, and $h=0$ or $|h| \geq 2 \pi / a$.

Suppose now that $h$ is real and $|h| \geq 2 \pi / a$. If

$$
\int_{-a}^{a} i t e^{i z t} d t=\int_{-a}^{a} e^{i z t}\left(e^{i h t}-1\right) f(t) d t
$$

for $f(t) \in L^{2}$, then uniqueness of Fourier transforms in $L^{2}$ ensures that

$$
f(t)=i t /\left(e^{i h t}-1\right)
$$

almost everywhere; but $f(t)$ is not in $L^{2}$ with the assumed condition on $h$. Thus the function on the left of $(22)$ is representable as $G^{\prime}(z)$ but not as $F(z+h)-$ $F(z)$. 


\section{REFERENCES}

1. R. P. Boas, Jr., Some theorems on fourier transforms and conjugate trigonometric integrals, Trans. Am. Math. Soc. (1936) p.298.

2. R. J. Duffin and A. C. Schaeffer, Some inequalities concerning entire functions of exponential type, Bulletin of Am. Math. Soc. (1937) p.554.

3. - Properties of functions of exponential type, Bull. Am. Math. Soc. (1938) p.236.

4. - On some inequalities of S. Bernstein and W. Markoff for derivatives of polynomials, Bull. Am. Math. Soc. (1938) p.289.

5. N. Levinson, Gap and density theorems, Am. Math. Soc. Col. Pub. Vol. XX VI, p.511 and 128.

6. R. E. A.C. Paley and N. Wiener, Fourier transforms in the complex domain, Amer. Math. Soc. Col. Pub. Vol. XIX, p.12 and 86-95.

7. M. Plancherel and G. Pólya, Fonctions Entieres et Integrales de Fourier Multiples, Comm. Math. Helv. 9 (1936-37), p. 224-248; 10 (1937-38), p. 110-163.

8. G. Pólya and G. Szegö, Aufgaben und Lehrsätze aus der Analysis, Dover Pub., Vol. I, p. 147, Problem 325.

University of California, Los Angeles 



\title{
PACIFIC JOURNAL OF MATHEMATICS
}

\author{
EDITORS
}

\author{
R. M. BOEINSON \\ University of California \\ Berkeley 4, California \\ E. HEWITT \\ University of Washington \\ Seattle 5, Washington
}

\author{
P. P. DILWOR TH \\ California Institute of Technology \\ Pasadena 4, California \\ E. F. BECKENBACH \\ University of California \\ Los Angeles 24, California
}

\section{ASSOCIATE EDITORSS}

$\begin{array}{llll}\text { H. BUSEMANN } & \text { P. R. HALMOS } & \text { BØRGE JESSEN } & \text { J. J. STOKER } \\ \text { HERBERT FFDERER } & \text { HEINZ HOPF } & \text { PAUL LÉVY } & \text { E. G. STR AUS } \\ \text { MARSHALL HALI } & \text { R. D. JAMES } & \text { GEORGE PÓLYA } & \text { KÔSAKU YOSIDA }\end{array}$

\section{SFONSORS}

UNIVERSITY OF BRITISH COLUMBIA

CALIFORNIA INSTITUTE OF TECHNOLOGY.

UNIVERSITY OF CAIIFORNIA, BERKELEY

UNIVERSITY OF CALIFORNIA, DAVIS

UNIVERSITY OF CALIFORNIA, LOS ANGELES

UNIVERSITY OF CALIFORNIA, SANTA BARBARA

UNIVERSITY OF NEVADA

OREGON STATE COLLEGE

UNIVERSITY OF OREGON

\author{
UNIVERSITY OF SOU THERN CALIFORNIA \\ STANFORD RESEARCH INSTITUTE \\ STANFORD UNIVERSITY. \\ WASHINGTON STATE COLLEGE \\ UNIVERSITY OF WASHINGTON \\ AMERICAN MATHEMATICAL SOCIETY \\ NATIONAL BUREAU OF STANDARDS, \\ INSTITUTE FOR NUMERICAL ANALYSIS
}

Mathematical papers intended for publication in the Pacific Journal of Mathematics should be typewritten (double spaced), and the author should keep a complete copy. Manuscripts may be sent to any of the editors-except Robinson, whose term expires with the completion of the present volume; they might also be sent to M.M. Schiffer, Stanford University, Stanford, California, who is succeeding Robinson. All other communications to the editors should be addressed to the managing editor, $\mathrm{E}$. F. Beckenbach, at the address given above.

Authors are entitled to receive 100 free reprints of their published papers and may obtain additional copies at cost.

The Pacific Journal of Mathematics is published quarterly, in March, June, September, and December. The price per volume (4 numbers) is $\$ 8.00$; single issues, $\$ 2.50$. Special price to individual faculty members of supporting institutions and to individual members of the American Mathematical Society: $\$ 4.00$ per volume; single issues, $\$ 1.25$.

Subscriptions, orders for back numbers, and changes of address should be sent to the publishers, University of California Press, Berkeley 4, California.

Printed at Ann Arbor, Michigan. Entered as second class matter at the Post Office, Berkeley, California.

\author{
UNIVERSITY OF CALIFORNIA PRESS - BERKELEY AND LOS ANGELES
}




\section{Pacific Journal of Mathematics}

\section{Vol. 3, No. $4 \quad$ June, 1953}

Paul Erdős and Gilbert Agnew Hunt, Jr., Changes of sign of sums of random variables........................................ 673

Paul Erdôs and Ernst Gabor Straus, On linear independence of sequences in

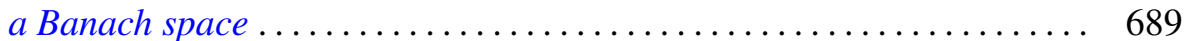

Haim Hanani, On sums of series of complex numbers ............... 695

Melvin Henriksen, On the prime ideals of the ring of entire functions ..... 711

Irving Kaplansky, Completely continuous normal operators with property L............................................. 721

Samuel Karlin, Some random walks arising in learning models. I. ...... 725

William Judson LeVeque, On uniform distribution modulo a subdivision ..................................... 757

Lee Lorch, Derivatives of infinte order ..................... 773

Ernest A. Michael, Some extension theorems for continuous functions . . . . . 789

Tyre Alexander Newton, A note on the Hölder mean ................ 807

Raymond Moos Redheffer, On a theorem of Plancherel and Pólya....... 823

Choy-Tak Taam, On the complex zeros of functions of Sturm-Liouville

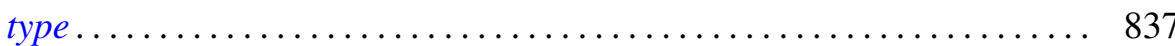

